\title{
Odontogenic cervical necrotizing fasciitis with descending mediastinitis: a case report and discussion
}

\author{
Arjunan Kumaran*, Xu Shuhui, Kang Wee Lee, Siti Radhziah
} Department of Otolaryngology, Division of Surgery \& Surgical Oncology, Singapore General Hospital, Singapore
Health Services, Outram Road, Singapore

Received: 26 October 2020

Accepted: 04 December 2020

\section{*Correspondence:}

Dr. Arjunan Kumaran,

E-mail: arjunan.kumaran@mohh.com.sg

Copyright: (c) the author(s), publisher and licensee Medip Academy. This is an open-access article distributed under the terms of the Creative Commons Attribution Non-Commercial License, which permits unrestricted non-commercial use, distribution, and reproduction in any medium, provided the original work is properly cited.

\begin{abstract}
$\mathrm{Mr} \mathrm{KE}$ is a 46 years old gentleman with a background of type 2 diabetes mellitus who was referred to our department for severe polymicrobial cervical necrotising fasciitis with descending mediastinitis after mandibular dental extraction. He was managed with endotracheal intubation, cervicotomy and video assisted thorascopic surgery (VATS), antibiotic and antifungal therapy and myocutaneous flap coverage. This case describes a classical presentation of an uncommon pathology and discusses options for airway management, surgical debridement, antimicrobial therapy and definitive defect coverage.
\end{abstract}

Keywords: Type 2 diabetes mellitus, VATS, Cervical Necrotising Fasciitis

\section{INTRODUCTION}

Cervical necrotising fasciitis (CNF) with descending necrotising mediastinitis (DNM) is rare but associated with significant morbidity and mortality. ${ }^{1}$ It most commonly occurs after mandibular dental extraction and begins as a submandibular abscess that extends to the para- and retropharyngeal spaces before descending along pre-vertebral fascial planes to the superior mediastinum. ${ }^{2}$ Less commonly this can occur from direct extension of tonsillar and peritonsillar infections, following trauma or surgery to the neck or after dental extractions in the maxilla which can also result in craniofacial necrotising fasciitis. ${ }^{3,4}$ Advanced age, presence of co-morbidity, involvement of two or more spaces (especially retropharynx) and presence of identifiable organism were predictive of progression of CNF to DNM. ${ }^{5}$

Treatment principles include (1) resuscitation and airway management, (2) aggressive (and repeated) surgical debridement, (3) broad-spectrum empirical followed by targeted antimicrobial therapy and (4) management of resultant defect and other complications. ${ }^{6}$ Typically, this entails a tracheostomy, debridement of the neck and chest, a combination of intravenous antibiotics and skin grafting. The authors describe a classical presentation of severe CNF with DNM that was instead managed with endotracheal intubation, cervicotomy and video assisted thorascopic surgery (VATS), additional antifungal therapy and myocutaneous flap coverage.

\section{CASE REPORT}

$\mathrm{Mr} \mathrm{KE}$ is a 46-year-old gentleman who was evacuated to our institution after failed treatment of odontogenic CNF with DNF in his home country. His past medical history includes hypertension and type 2 diabetes mellitus.

Mr E underwent a left mandibular molar extraction which was complicated by a non-resolving submandibular abscess despite traditional chinese herbal medications and oral antibiotics. He was admitted to hospital, given intravenous antibiotics and underwent incision and drainage with insertion of passive drains. Unfortunately, 
his condition deteriorated and he suffered a cardiopulmonary collapse. He was resuscitated and transferred to our institution for further care ( 8 days after his dental extraction).

On arrival to the emergency Department, $\mathrm{Mr} \mathrm{E}$ was febrile and drowsy with a glasgow coma score (GCS) of 9 (E3V2M4). 4 passive drains over his neck (3) and chest (1) and 3 intra-oral (right buccal mucosa, retromolar trigone and floor of mouth) incisions were seen from which purulent discharge was freely draining (Figure 1). Crepitus was felt over his neck and chest and on nasoendoscopy, his right tonsil appeared unhealthy and parapharyngeal walls edematous but airway was patent.
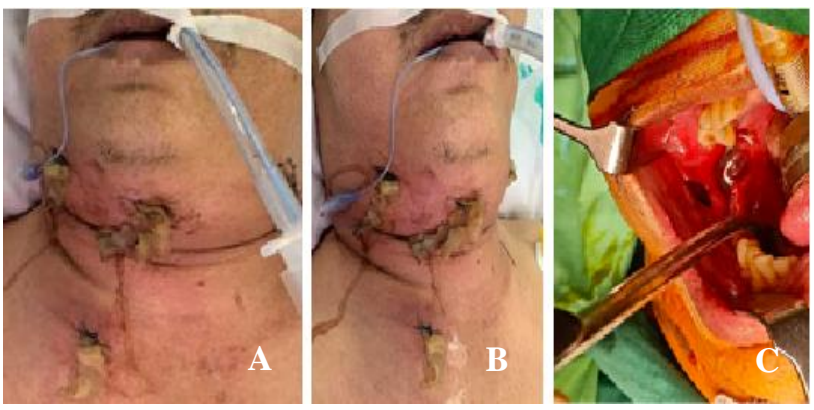

Figure 1: Intra-operative photographs demonstrating

(A) the neck (B) chest (C) intra-oral incisions.

Biochemical investigations revealed an elevated total white count $\left(26.52 \times 10^{\wedge} 9 / \mathrm{L}\right)$, C-reactive peptide $(265$ $\mathrm{mg} / \mathrm{L})$, serum glucose $(17.1 \mathrm{mmol} / \mathrm{L})$, ketones $(0.1$ $\mathrm{mmol} / \mathrm{L})$ and lactate $(5.4 \mathrm{mmol} / \mathrm{L})$. Platelets were not low
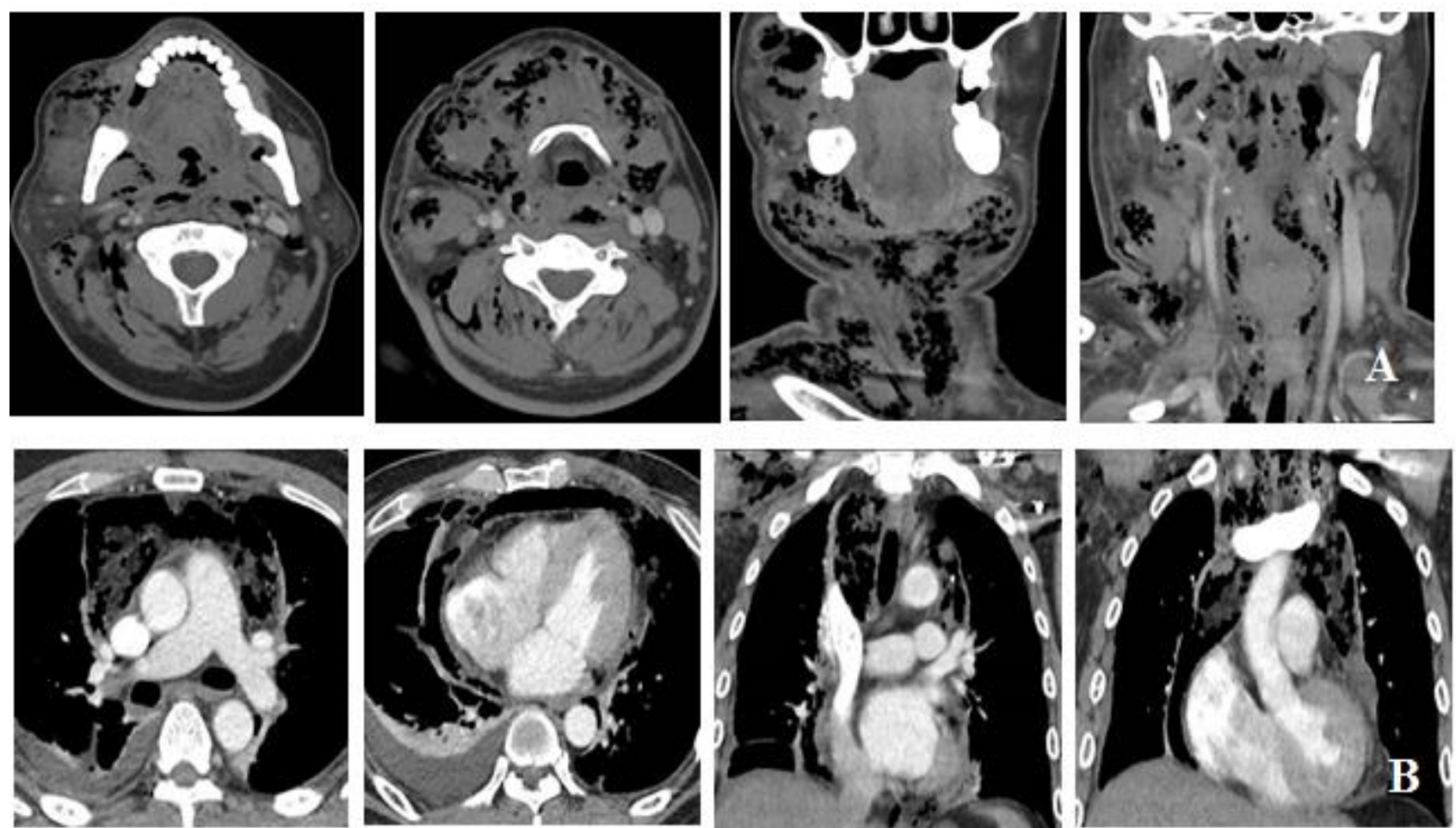

Figure 2: (A) Lateral neck (B) Chest X-radiographs
demonstrating extensive cervical and mediastinal

Figure 2: (A) Lateral neck (B) Chest X-radiograph
demonstrating extensive cervical and mediastinal emphysema.

Computed tomography (CT) scans of the neck and chest localised this to bilateral submandibular, retropharyngeal and prevertebral spaces with caudal extension to involve the mediastinum down to the level of the oesophageal hiatus (Figure 3).

$\left(170 \times 10^{\wedge} 9 / \mathrm{L}\right)$ but coagulation was prolonged (prothrombin time $14.1 \mathrm{~s}$ and activated partial thromboplastin time $33.3 \mathrm{~s})$. Serum bicarbonate (26.7

Lateral neck and chest $\mathrm{X}$ radiographs demonstrated extensive subcutaneous and mediastinal soft tissue gas suggestive of necrotizing fasciitis (Figure 2).

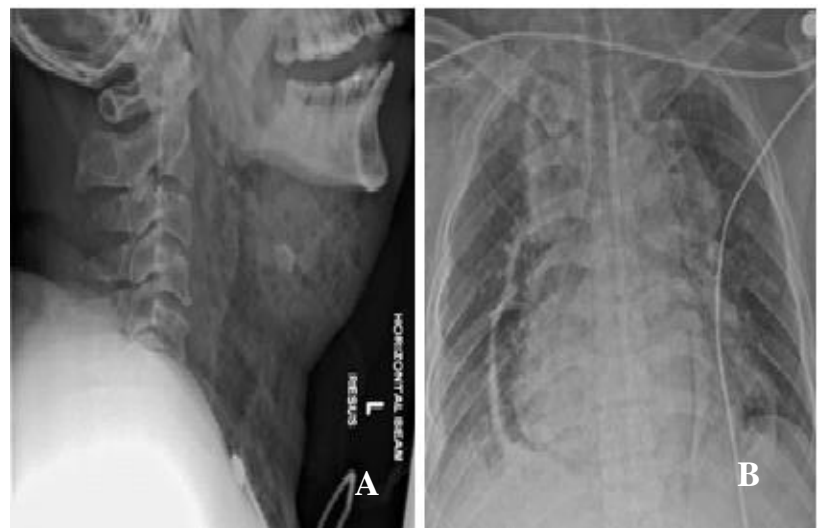

Figure 3: (A) axial and coronal CT images demonstrating the locations of abscesses in the neck (B) axial and coronal CT images demonstrating the locations of abscesses in the chest. 
While in the Emergency Department, Mr E developed fast atrial fibrillation and septic shock that was treated with supplemental oxygen through a face mask, intravenous fluid resuscitation and an anti-arrhythmic infusion. He was given empirical intravenous antibiotics which comprised penicillin G, vancomycin and clindamycin before undergoing emergency surgical debridement.

He was successfully intubated awake with an armored endotracheal tube using a fiber optic bronchoscope and debridement sequentially performed by oral and maxillofacial surgery (OMS), otolaryngology (ENT) and cardiothoracic surgery (CTS) under general anesthesia. Complete \#38 lingual wall loss, visualisation of inferior alveolar nerve and expression of purulent material through the socket confirmed the source of the infection. Turbid dishwater fluid was seen in the right submandibular, bilateral parapharyngeal and retropharyngeal spaces and right sternohyoid, strenothyroid and sternocleidomastoid muscles were necrotic but carotid sheaths were intact. Mostly serous effusions in loculated pockets with no gross purulent material was encountered on VATS. Intra-operative hypotension necessitated dual agent inotropic support and $\mathrm{Mr} \mathrm{E}$ was transferred to the intensive care unit (ICU) after.

In the ICU, he developed acute kidney injury requiring temporary continuous renal replacement therapy, inotropic support was weaned off he was extubated successfully after 7 days and transferred to high dependency.
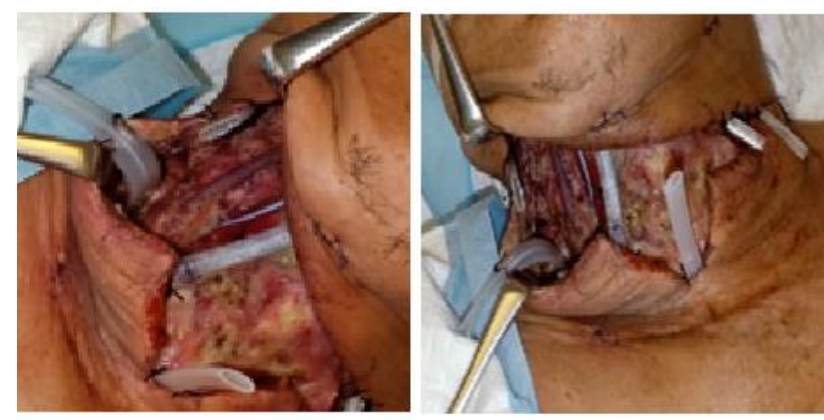

Figure 4: Intra-operative photographs demonstrating the depth of dissection and use of multiple passive drains.

Initial intra-operative samples taken for microscopy reported the presence of gram-positive cocci and bacilli and gram-negative bacilli. Bacterial cultures of these samples yielded Streptococcus anginosus, Bacteroides pyogenes and Prevotella Intermedia while both acid-fast bacilli and fungal cultures were negative. Mr E required a total of 6 debridements in operating theatre (Figure 4) and further samples were taken for repeat cultures which then grew Candida albicans, Klebsiella pneumonia and
Corynebacterium for which intravenous Anidulafungin and Meropenam were added.

Mr E's clinical course was stormy and complicated by a bleeding duodenal ulcer that required repeat endoscopic therapy and mesenteric angioembolisation and lower limb deep venous thrombosis for which an inferior vena cava filter was inserted and anticoagulation started. Despite this, with regular bedside debridement and negative pressure wound therapy with vacuum assisted closure (VAC) dressing, he made a good recovery. Chest tubes were sequentially removed and he was transferred well from high dependency and to the general ward.

He finally underwent a pectoralis major flap reconstruction for coverage of his anterior neck defect (Figure 5). This was complicated by a small inferior wound dehiscence managed conservatively with regular VAC dressing changes and $\mathrm{Mr} \mathrm{E}$ was discharged well after 88 days.

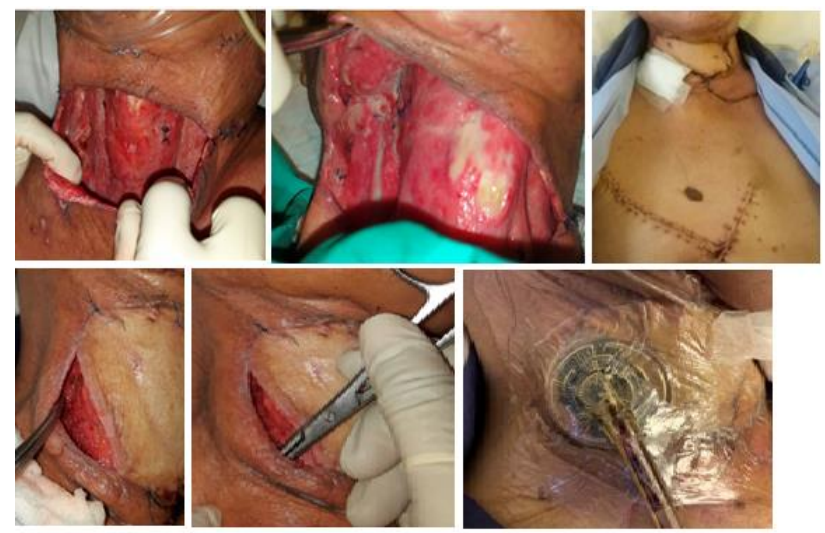

Figure 5: Resultant neck defect, pectoralis major flap reconstruction and management of small dehiscence with VAC.

\section{DISCUSSION}

\section{Airway management}

In the operating theatre, awake fiber optic bronchoscopic endotracheal intubation was performed successfully with ENT on standby for an emergency primary tracheostomy in case of airway loss. As $\mathrm{Mr} \mathrm{E}$ was extubated successfully 7 days later, he did not require a secondary tracheostomy.

Tracheostomy under local anaesthesia has been the gold standard of airway management in deep neck space infections. ${ }^{6}$ Unlike endotracheal intubation, it reduces the need for sedation, allows earlier step-down from ICU, avoids subglottic stenosis and facilitates bronchopulmonary toilet. In addition, early tracheostomy within 7 days may reduce need for mechanical ventilation and thereby length of ICU stays and overall healthcare costs. ${ }^{7}$ However, primary tracheostomy in this setting may prove especially technically challenging in a 
compromised airway with distorted anatomy with risk of seeding the infection into the airway and mediastinum. ${ }^{8}$

In CNF, endotracheal intubation may also be difficult as trismus may limit mouth opening and abscess mass may restrict neck extension. However, with newer technologies and techniques such as video laryngoscopy and awake fiber optic intubation, the larynx can be visualized without aligning the oral, pharyngeal and laryngeal axes. $^{8}$ Awake intubation also offers an additional benefit of cardiovascular stability which is important in cases of septic shock. ${ }^{9}$

\section{Surgical debridement and coverage}

Classically, bilateral cervicotomy via Lahey hockey stick incisions, subplatysmal flap elevation and debridement of necrotic tissue has been described in CNF. ${ }^{6}$ This was performed for $\mathrm{Mr} \mathrm{E}$, after which several passive drains were inserted and neck wound packed.

After repeated debridement, bilateral sternohyoid, sternothyroid and sternocleidomastoid muscles, bilateral parotid tails and part of the left submandibular gland were removed with bilateral carotid sheaths left intact. As this defect was large and deep a pectoralis major flap was raised for coverage. A small area of dehiscence was managed conservatively with negative pressure therapy which has been shown to control infections and facilitate wound bed granulation. ${ }^{10}$

As for the mediastinum in DNM, Endo et all divided it into three sections, I: above carina, IIa: below carina and anterior, IIb: below carina and both anterior and posterior and drainage is performed transcervical in type I, subxiphoid or with a sternotomy in type IIa and with a thoracotomy in type IIb. ${ }^{11}$

Mr E had type IIb DNM but underwent VATS instead of a thoracotomy. A bronchial blocker was placed, $\mathrm{Mr} \mathrm{E}$ turned lateral and two VATS incisions made. The extrapleural plane was entered and extended to the anterior mediastinum and decortication performed till lung expansion adequate. Two chest tubes were inserted and wounds closed. VATS was technically feasible but periods of apnea with lung isolation and hypotension on turning lateral (possibly due to compression of great vessels) were observed.

It may not be routine but in centers with expertise and equipment for VATS, it can offer a minimally invasive option for mediastinal drainage in patients unfit for radical surgery even in extensive subcarinal disease (type II DNM). ${ }^{12,13}$

\section{Antimicrobial therapy}

Though often polymicrobial, gram positive bacteria are most commonly identified with Streptococcus, Staphylococcus and Prevotella species predominating. ${ }^{1,4,14}$ These may occur together with anaerobes as well as gram negative bacteria especially in diabetic patients. ${ }^{5,15}$ Fungal co-infection in DNM is less common and has only been described twice, one which occurred primarily as a deep neck space infection and another that developed from a surgical site infection after total thyroidectomy. ${ }^{16,17}$

Empirical antibiotics for necrotising fasciitis (regardless of location) in our institution includes penicillin G, vancomycin and clindamycin which was adequate to treat the bacteria isolated from initial cultures but repeated cultures should be routinely taken to check for other pathogens and secondary infections. Vigilance should be practiced to periodically update empirical antibiotic regimes to keep up with changing microbial trends. ${ }^{18}$

\section{Multi-disciplinary care}

Mr E, like many patients with CNF and DNM developed atrial fibrillation and septic shock which required fluid resuscitation, anti-arrythmics and inotropic support in the Emergency Department. This was led by the Emergency Physicians and surgical teams with assistance from the Anaesthesia and ICU Team.

During his hospital stay, he was also reviewed by Gastroenterologists and interventional radiologists for a bleeding duodenal ulcer as well as a Haematologist for management of deep venous thrombosis of the lower limb. An infectious disease specialist supervised the antimicrobial therapy while the various Surgical teams performed the debridements and dressing changes.

As part of his recovery, he received enteral nutritional support with the help of a dietician and speech therapist, mobilisation and strengthening exercises for deconditioning from physiotherapists and constant nursing care for his wounds, dressing and drains. This highlights the need for multi-disciplinary in the care of patients with CNF and DNM.

Due to the prolonged hospital stay and multiple procedures, cost of care for Mr E was very high and a social worker was involved. Thus, even though hyperbaric oxygen therapy has been shown to be beneficial in CNF with DNM and was offered to Mr E, it was ultimately declined due to cost. ${ }^{19}$ In a study of patients with CNF in Tanzania, $88.1 \%$ of patients delayed seeking treatment as there was no such service available, they could not afford it, they chose to try traditional healing or services were too far from their residence. This represents an area of concern as patients with poorer socioeconomic support may not be able to afford timely and appropriate treatment. ${ }^{20}$

\section{CONCLUSION}

Awake fiber optic bronchoscopic endotracheal intubation with early extubation offers a less morbid alternative to 
tracheostomy. VATS is an effective sternotomy and thoracotomy sparing approach to mediastinal drainage even with extensive involvement. Though uncommon, fungal infections can co-exist in CNF. Routine empirical antifungals are not required. With deeper neck debridement, a pectoralis major myocutaneous flap is a good option for reconstruction. Prophylaxis for PUD and DVT should be considered given repeated surgical debridement and prolonged hospital stay CNF with DNM requires early multi-disciplinary care by physicians, surgeons, anesthetists, nurses, dieticians, speech therapists, physiotherapists and social workers.

\section{Funding: No funding sources \\ Conflict of interest: None declared \\ Ethical approval: Not required}

\section{REFERENCES}

1. Tapiovaara L, Bäck L, Aro K. Comparison of intubation and tracheotomy in patients with deep neck infection. Eur Arch Otorhinolaryngol. 2017;274(10):3767-72.

2. Gunaratne DA, Tseros EA, Hasan Z. Cervical necrotizing fasciitis: Systematic review and analysis of 1235 reported cases from the literature. Head Neck. 2018;40(9):2094-102.

3. Suárez A, Vicente M, Tomás JA, Floría LM, Delhom J, Baquero MC. Cervical necrotizing fasciitis of nonodontogenic origin: case report and review of literature. Am $\mathrm{J}$ Emerg Medic. 2014;32(11):1441-e5.

4. Mtenga AA, Kalyanyama BM, Owibingire SS, Sohal KS, Simon ENM. Cervicofacial necrotizing fasciitis among patients attending the Muhimbili National Hospital, Dar es Salaam, Tanzania. Bio Med Cent Infect Dis. 2019;19(1):642.

5. Kang SK, Lee S, Oh HK, Kang MW, Na MH, Yu $\mathrm{JH}$, et al. Clinical features of deep neck infections and predisposing factors for mediastinal extension. Korean J Thorac Cardiovasc Surg. 2012;45(3):171.

6. Osborn TM, Assael LA, Bell RB. Deep space neck infection: principles of surgical management. Oral Maxillofac Surg Clin North Am. 2008;20(3):35365.

7. Griffiths J, Barber VS, Morgan L, Young JD. Systematic review and meta-analysis of studies of the timing of tracheostomy in adult patients undergoing artificial ventilation. Bio Med J. 2005;330:1243.

8. Chen SJ, Ji N, Chen YX, Zhao SJ, Xiao JR, Lin $\mathrm{XM}$, et al. Management and maintenance of the airway in cervical necrotising fasciitis: a retrospective analysis of 15 cases. $\mathrm{Br} \mathrm{J}$ Oral Maxillofac Surg. 2015;53(7):642-6.

9. Machata AM, Gonano C, Holzer A, Andel D, Spiss $\mathrm{CK}$, Zimpfer $\mathrm{M}$, et al. Awake nasotracheal fiberoptic intubation: patient comfort, intubating conditions, and hemodynamic stability during conscious sedation with remifentanil. Anesthes Analges. 2003;97(3):904-8.

10. Frankel JK, Rezaee RP, Harvey DJ, McBeath ER, Zender CA, Lavertu P. Use of negative pressure wound therapy with instillation in the management of cervical necrotizing fasciitis. Head Neck. 2015;37(11):E157-60.

11. Endo S, Murayama F, Hasegawa T, Yamamoto S, Yamaguchi T, Sohara Y, et al. Guideline of surgical management based on diffusion of descending necrotizing mediastinitis. Japan J Thorac Cardiovasc Surg. 1999;47(1):14-9.

12. Suga A, Inoue $Y$, Takeichi H, Yamada S, Iwazaki M. A case of an elderly patient treated for descending necrotizing mediastinitis. Gen Thorac Cardiovasc Surg. 2011;59(9):623-6.

13. Chen KC, Chen JS, Kuo SW, Huang PM, Hsu HH, Lee JM, et al. Descending necrotizing mediastinitis: a 10-year surgical experience in a single institution. J Thorac Cardiovasc Surg. 2008;136(1):191-8.

14. Parhiscar A, Har-El G. Deep neck abscess: a retrospective review of 210 cases. Ann Otol Rhinol Laryngol. 2001;110:1051-4.

15. Adekanye AG, Umana AN, Offiong ME, Mgbe RB, Owughalu BC, Inyama $M$, et al. Cervical necrotizing fasciitis: management challenges in poor resource environment. European Arch Oto-RhinoLaryngol. 2016;273(9):2779-84.

16. Abbasi Z, Inam $H$, Das $S$, Neel $S$, Fatimi $S H$. Fungal Cervical Abscess Complicated by Necrotizing Fasciitis Leading to Descending Necrotizing Mediastinitis: A Case Report. Cureus. 2019;11(8):e5369.

17. Aslier M. Necrotizing Fasciitis due to Candida Infection after Thyroid Surgery. Turk Arch Otorhinolaryngol. 2020;58(1):56-60.

18. Sethi DS, Stanley RE. Deep neck abscesses changing trends. J Laryngol Otol. 1994;108:138-43.

19. Elander J, Nekludov M, Larsson A, Nordlander B, Eksborg S, Hydman J. Cervical necrotizing fasciitis: descriptive, retrospective analysis of 59 cases treated at a single center. Eur Arch Otorhinolaryngol. 2016;273(12):4461-7.

20. Gore MR. Odontogenic necrotizing fasciitis: a systematic review of the literature. Bio Med Cent Ear Nose Throat Disord. 2018;18:14.

Cite this article as: Kumaran A, Shuhui X, Lee KW, Radhziah S. Odontogenic cervical necrotizing fasciitis with descending mediastinitis: a case report and discussion. Int J Otorhinolaryngol Head Neck Surg 2021;7:143-7. 